syphilis may lead to more serious complications in this population. We sought to inductively explore syphilis-related knowledge, and attitudes around biomedical prevention options for syphilis in an age of HIV pre-exposure prophylaxis (PrEP), with the goal of informing effective strategies to address the syphilis epidemic.

Methods We conducted in-depth, one-on-one interviews with a heterogeneous sample of GBM in Vancouver, including men living with HIV and/or with a history of syphilis. Our interviews focused on participants' knowledge around syphilis and perceptions regarding syphilis PrEP. Interviews were audiorecorded, transcribed verbatim, and analyzed using Grounded Theory.

Results Twenty-five GBM were interviewed (64\% white; median age: 43 years). Four overarching themes emerged regarding men's views about syphilis. First, syphilis-related knowledge differed according to HIV and syphilis serostatus. Second, competing ideas emerged regarding men's concerns about syphilis. While our participants expressed concern about getting syphilis, they also described the importance of sexual intimacy and pleasure. Third, many participants said that syphilis was not perceived to be particularly alarming; preventing HIV infection remained a primary concern for many. Finally, although syphilis PrEP was appealing to some, participants were concerned about antibiotic resistance, cost, and side effects.

Conclusion Concern for syphilis appeared low among GBM. Our participants tended to organize their safer sex strategies around HIV, not syphilis. Although syphilis-related knowledge was relatively high among GBM living with HIV and those with a prior syphilis diagnosis, this knowledge did not appear to be associated with safer sexual practices, such as increased condom use. This work highlights the importance of examining other potential acceptable prevention solutions, such as syphilis PrEP.

Disclosure No significant relationships.

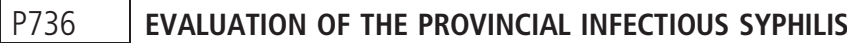 PARTNER NOTIFICATION PROGRAM IN BRITISH COLUMBIA, CANADA}

${ }^{1}$ Christine Lukac*, ${ }^{2}$ Theodora Consolacion, ${ }^{2}$ Venessa Ryan, ${ }^{2}$ Emma Cumming, ${ }^{2}$ Geoffrey Ford, ${ }^{1}$ Gina Ogilvie, ${ }^{2}$ Mark Gilbert, ${ }^{2}$ Troy Grennan, ${ }^{2} J a s o n$ Wong. ${ }^{1}$ University of British Columbia, Faculty of Medicine, Vancouver, Canada; ${ }^{2} B C$ Centre for Disease Control, Clinical Prevention Services, Vancouver, Canada

\subsection{6/sextrans-2019-sti.796}

Background Infectious syphilis partner notification $(\mathrm{PN})$ is centrally coordinated at the British Columbia (BC) Centre for Disease Control. Approaches include patient-initiated and provider-initiated $\mathrm{PN}$, and outcomes include the proportion of partners (1) notified of possible exposure to syphilis, (2) tested and/or treated, and (3) diagnosed. Among gay, bisexual and other men who have sex with men (gbMSM) who have the greatest burden of syphilis, we evaluated PN outcomes between patient-initiated and provider-initiated PN.

Methods All infectious syphilis diagnoses in 2016 in BC were included. Syphilis re-diagnosis was defined as a syphilis diagnosis in 2016 with at least one additional diagnosis in 20062016, while first-diagnosis was defined as a syphilis diagnosis in 2016 only. PN outcomes were calculated along a cascadeof-care framework, where the numerator is the denominator of the subsequent indicator. Chi-square tests compared PN outcomes of patient-initiated versus provider-initiated PN, within strata of gbMSM first-diagnosed and re-diagnosed.

Results Of the 759 infectious syphilis cases in BC in 2016, 648 (85\%) were among gbMSM, among whom 474 (73\%) were first-diagnoses and 174 (27\%) were re-diagnoses. A significantly greater proportion of gbMSM first-diagnosed chose patient-initiated PN compared to gbMSM re-diagnosed $(62 \%$ vs $42 \% ; \mathrm{P}<0.01)$. Among gbMSM first-diagnosed, patient-initiated $\mathrm{PN}$ resulted in a greater proportion of partners notified compared to provider-initiated PN (177/199; 89\% vs 426/603; $70 \% ; \mathrm{P}<0.001)$. There was no difference in the proportion of partners tested and/or treated, $(156 / 177 ; 88 \%$ vs $380 / 426$; $89 \% ; \mathrm{P} \geq 0.05)$, and diagnosed (24/156 15\% vs 51/380 13\%; $\mathrm{P} \geq 0.05)$. A similar trend in $\mathrm{PN}$ outcomes was observed among partners of gbMSM re-diagnosed.

Conclusion Patient-initiated and provider-initiated PN had similar outcomes among partners of both gbMSM first-diagnosed and re-diagnosed. However, gbMSM first-diagnosed were more likely to choose to notify their own partners. These findings demonstrate that patient-initiated $\mathrm{PN}$ have similar outcomes to provider-initiated $\mathrm{PN}$ and can increase the overall capacity for PN.

Disclosure No significant relationships.

\section{P737 EVALUATING SYPHILIS PARTNER NOTIFICATION OUTCOMES IN SEVEN JURISDICTIONS}

${ }^{1}$ Anna Cope*, ${ }^{2}$ James Matthias, ${ }^{3}$ Mohammad Rahman, ${ }^{4}$ Jill Diesel, ${ }^{5}$ River Pugsley, ${ }^{6}$ Julia Schillinger, ${ }^{7}$ Rilene $\mathrm{Ng},{ }^{8}$ Ellen Klingler, ${ }^{9}$ Victoria Mobley, ${ }^{9}$ Erika Samoff, ${ }^{10}$ Kyle Bernstein, ${ }^{11}$ Thomas Peterman. ${ }^{1}$ Centers for Disease Control and Prevention, Division of STD Prevention, Raleigh, USA; ${ }^{2}$ Centers for Disease Control and Prevention, Division of STD Prevention, Tallahassee, USA; ${ }^{3}$ Centers for Disease Control and Prevention, Division of STD Prevention, New Orleans, USA; ${ }^{4} C D C$, MDHHS, NCHHSTP, DSTDP, STD Section, Detroit, USA; ${ }^{5}$ Centers for Disease Control and Prevention, Division of STD Prevention, Richmond, USA; ${ }^{6}$ Centers for Disease Control and Prevention, Division of STD Prevention, New York City, USA; ${ }^{7}$ Centers for Disease Control and Prevention, Division of STD Prevention, San Francisco, USA; ${ }^{8}$ New York City Department of Health and Mental Hygiene, New York City, USA; ${ }^{9}$ North Carolina Division of Public Health, Communicable Disease Branch, Raleigh, USA; ${ }^{10}$ Centers for Disease Control and Prevention, Atlanta, USA; ${ }^{11}$ Centers for Disease Control and Prevention, Division of STD Prevention, Atlanta, USA

10.1136/sextrans-2019-sti.797

Background The effectiveness of partner notification services (PNS) for limiting syphilis transmission relies on the ability of disease intervention specialists (DIS) to find and assure treatment of partners. We measured estimates of partners found and treated due to PNS in seven jurisdictions.

Methods We reviewed early syphilis cases (primary, secondary, early latent) reported during 2015-2017 in seven jurisdictions in the United States (Florida, Louisiana, Michigan, North Carolina, Virginia, New York City, and San Francisco). We measured the numbers of: early syphilis (index) cases interviewed by DIS, (sex) partners reported (primary cases: $\leq 3$ months; secondary cases: $\leq 6$ months; early latent cases: $\leq 1$ year), partners with enough locating information to begin PNS, partners treated prophylactically, and infected partners brought in for treatment resulting from PNS. We considered partners to be brought to treatment by PNS if: 1) a DIS-assigned disposition code indicated "brought to treatment" or 2) the partner was treated 0-90 days after the index case was interviewed.

Results DIS interviewed 23,428 index patients with early syphilis (range among jurisdictions 1,106-9,388), representing $78.9 \%$ of reported cases $(50.1 \%-99.5 \%)$. Of those interviewed, $18,482(78.9 \%)$ reported 78,960 partners, of whom 
20,771 (26.3\%) had enough locating information to begin PNS. Among these partners initiated for PNS, 5,851 were unlocatable/refused PNS (28.2\%, range: 23.9\%-38.8\%), 5,959 were prophylactically treated (28.6\%, range: $2.1 \%-39.8 \%)$ and 5,905 were classified as infected and brought to treatment (28.4\%; range: $12.1 \%-37.3 \%)$. After excluding partners treated before $(n=1,436)$ and $\geq 90$ days after $(n=90)$ the index case interview, 4,379 partners were considered infected and brought to treatment $(0.15$ partners per reported case [range $0.02-0.50$ ] or 0.18 partners per interviewed case [range $0.05-0.60])$.

Conclusion For every 5 to 6 index patients interviewed, PNS resulted in 1 infected partner brought to treatment. The success of DIS in finding and bringing partners to treatment varied across jurisdictions.

Disclosure No significant relationships.

\section{P738 NO BEJEL AMONG TREPONEMA PALLIDUM ISOLATES DIAGNOSED AS SYPHILIS FROM SURINAM, ANTILLEAN AND DUTCH CLIENTS IN AMSTERDAM}

${ }^{1}$ Helene Zondag*, ${ }^{2}$ Sylvia Bruisten, ${ }^{3}$ Eliška Vrbová, ${ }^{3}$ David Šmajs. ${ }^{1}$ Public Health Service Amsterdam, Infectious Diseases, Public Health Laboratory, Amsterdam, Netherlands; ${ }^{2}$ Public Health Service Amsterdam, Amsterdam University Medical Center (UMC), Infectious Diseases, Infection and Immunity (AI and II), Amsterdam, Netherlands; ${ }^{3}$ Masaryk University, Department Of Biology, Brno, Czech Republic

\subsection{6/sextrans-2019-sti.798}

Background Treponema pallidum subsp. pallidum (TPA) is the causative agent of syphilis, a world-wide prevalent venereal disease. Bejel is caused by $T$. pallidum subsp. endemicum (TEN), which shows similar clinical manifestations and is morphologically and serologically indistinguishable from TPA. The PCR used for syphilis diagnostics, targeting the polA gene, does not discriminate between subspecies of T. pallidum. Bejel is thought to be restricted to semi-arid areas and its transmission to be non-venereal, but recently, in patients diagnosed with syphilis in Cuba and Japan, sexual transmission of TEN was shown to occur. We therefore performed molecular typing on samples from Surinam, Antillean and Dutch patients to discover bejel causing TEN strains among syphilis cases in Amsterdam.

Methods DNA was extracted from 137 ulcer swabs collected between 2006 and 2018 from male clients attending the Amsterdam sexually transmitted infections (STI) clinic. MLST was performed by partial sequence analysis of the $t p 0136$, tp0548 and tp0705 genes to generate allelic profiles. In addition, 23S rRNA loci were checked for A2058G and A2059G macrolide resistance mutations.

Results We found 15 distinct allelic profiles from 99/137 (72\%) fully typed samples, of which none were TEN, $83 \%$ were SS14-like strains and $17 \%$ Nichols-like. The most prevalent types were 1-3-1 (44\%) and 1-1-1 (19\%), in concordance with similar TPA typing studies. There was no association found between TPA types and ethnicity. Five new allelic types and profiles were found adding to the knowledge of TPA strain diversity. The successfully sequenced $23 \mathrm{~S}$ rRNA loci from 123/137 (90\%) samples showed the presence of A2058G and A2059G mutations, 79\% and 2\% respectively.

Conclusion No misdiagnoses were found within the samples from different ethnicities residing in Amsterdam, the
Netherlands. The strain diversity found in this study reflects the local male STI clinic population which is a diverse, mixed group.

Disclosure No significant relationships.

\section{P739 NOVEL RAPID TEST FOR IMPROVED DIAGNOSIS OF ACTIVE SYPHILIS AT THE POINT OF CARE}

${ }^{1}$ Minh Pham*, ${ }^{2}$ Mary Garcia, ${ }^{2}$ Huy Van, ${ }^{3}$ Karl Technau, ${ }^{3}$ Amy Wise, ${ }^{2}$ David Anderson. ${ }^{1}$ Burnet Institute, Public Health, Melbourne, Australia; ${ }^{2}$ Burnet Institute, Global Health Diagnostics, Melbourne, Australia; ${ }^{3}$ Rahima Moosa Mother and Child Hospital, Johannesburg, South Africa

\subsection{6/sextrans-2019-sti.799}

Background Syphilis has been and still is one of the greatest global health concerns. Syphilis can seriously damage the nervous system of infected individuals including infants born to infected mother. Treatment of syphilis is simple and effective with penicillin but diagnosis is challenging, particularly in resource-constrained settings, due to the need for a laboratory-based confirmatory test. Current point of care (POC) tests for syphilis are available but cannot distinguish active infections from past treated infections with a misclassification rate of up to $50 \%$ (low specificity). We developed a prototype rapid POC test (IgA Confirm) that can differentiate active syphilis from past treated infections at the point of care.

Methods We conducted a prospective diagnostic accuracy study to assess the specificity (and sensitivity) of the IgA Confirm test in identifying active syphilis infections classified by Treponema pallidum Antibobody (TPAb) and rapid plasma regain (RPR) laboratory serology. Between June-December 2018, 500 pregnant women attending Rahima Moosa Mother and Child hospital, South Africa were recruited and provided venous blood samples for syphilis testing including the IgA Confirm (index) and laboratory serology (reference) tests.

Results The IgA Confirm demonstrate a sensitivity of $100 \%$ $(5 / 5)$ for identifying samples with active syphilis infections (TPAb positive and RPR positive); 100\% (9/9) specificity for identifying samples with past or treated infections (TPAb positive, RPR negative) and, 99.4\% (484/487) specificity for samples with no evidence of syphilis (TPAb and RPR negative). Conclusion This study showed that the IgA Confirm test has the ability to identify active syphilis infection and meet the WHO Target Product Profile for syphilis confirmatory testing. Future study is needed to further evaluate diagnostic performance of the test in high prevalence setting.

Disclosure No significant relationships.

\section{P740 IMPROVING SYPHILIS DIAGNOSIS AND TREATMENT IN AN URBAN POPULATION THROUGH ROUTINE EMERGENCY DEPARTMENT SCREENING}

Kimberly Stanford*, Jessica Ridgway, John Schneider. University of Chicago, Medicine, Chicago, USA

\subsection{6/sextrans-2019-sti.800}

Background With the recent nationwide increase in syphilis, it is imperative to find novel means of reaching at-risk populations for early diagnosis and treatment. Many urban communities have both high rates of syphilis and frequently utilize the 